\title{
Stagnation-point flow and heat transfer over an exponentially stretching/shrinking
} cylinder

\begin{abstract}
The stagnation-point boundary-layer flow and heat transfer over an exponentially stretching/shrinking cylinder is studied. The governing partial differential equations in cylindrical form are first transformed into ordinary differential equations which are then solved numerically using a shooting method. Results for the skin friction coefficient, local Nusselt number, velocity and temperature profiles are presented and discussed in detail. The effects of the stretching/shrinking parameter $\varepsilon$ and the curvature parameter $\gamma$ on the fluid flow and heat transfer characteristics are also examined. It is found that the solutions for a shrinking cylinder are non-unique and that the surface shear stress and the heat transfer rate at the surface increase as the curvature parameter increases.
\end{abstract}

Keyword: Stagnation-point flow; Exponentially stretching/shrinking cylinder; Heat transfer; Dual solutions 
